Proc. Indian Acad. Sci. (Chem. Sci.), Vol. 107, No. 2, April 1995, pp. 109-122.

(C) Printed in India.

\title{
A one-step eficient general method for the synthesis of naphthopyrones: A first synthesis of spiro [naphthalene-2, 4'-phenanthrene] carbocycles
}

\author{
JAYSUKHLAL R MERCHANT* and NEVILLE V FERNANDES \\ Organic Chemistry Research Department, D G Ruparel College, Senapati Bapat \\ Marg, Bombay 400016, India
}

MS received 18 April 1994; revised 26 September 1994

\begin{abstract}
Abetract. A new synthesis of naphthopyrones and hexahydrospiro[naphthalene2,4'-phenanthrene]-1,1'-diones has been accomplished by the Friedel-Crafts reaction of various 1-tetralones with $\alpha, \beta$-unsaturated acids in the presence of polyphosphoric acid (PPA).
\end{abstract}

Keywords. Tetralones; $\alpha, \beta$-unsaturated acids; $4 \mathrm{H}$-naphtho(1,2-b)pyrone; spiro [naphthalene-2, 4'-phenanthrene]-1,1'-dione.

\section{Introduction}

Naphthopyrones and related $o$-heterocycles form an interesting class of compounds. This is mainly due to their widespread occurrence in nature (Dean 1963; Kulkarni et al 1972) and the number of biological activities they exhibit. For example, $\beta$-lapachone (1) and a number of its synthetic derivatives (Schaffner-Sabba et al 1984) have been shown to be active against the retrovirus reverse transcriptase. Further, a new series of antiallergic compounds(Nohara et al 1975, 1977)(2) possessing naphthopyrone moiety has been reported. Apart from these, other biological effects such as anticonvulsant (Ambrogini and Passerini 1976) and antitubercular (Annigeri et al 1966) activities have been reported (Ronald 1975).

It can thus be seen that naphthopyrones are important intermediates in the synthesis of many naturally occurring and pharmacologically important compounds. A survey of literature reveals that this ring system could be synthesised by methods which either involve a number of steps (von Strandtmann et al 1972) or give a mixture of products (Schafiner-Sabba et al 1984). Therefore a simple and practical method for their synthesis is highly desirable. With this in mind we sought to develop a new method for the synthesis of this class of compounds from readily available starting materials. The envisioned route, as evident from the retrosynthetic perspective, basically involves condensation of enolisable aromatic ketones, such as 1-tetralone, with $\alpha, \beta$-unsaturated acids in the presence of an acid, e.g. polyphosphoric acid (PPA).

\section{Reenlts and discresion}

To begin with we reacted 1-tetralone (1 eq.) with acrylic acid (2 eq.) in the presence of freshly prepared PPA at $125^{\circ} \mathrm{C}$. The reaction afforded three products (scheme 1) after

\footnotetext{
- For correspondence
} 
<smiles>[R]c1cc([R6])c2c(c1[R5])C(=O)CCC2[R2]</smiles>

$\underline{3}$

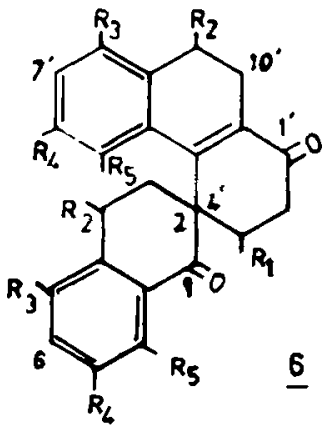

a $: R_{1}-R_{5}=H$

$b=R_{2}-R_{5}=H_{1}, R_{1}=\mathrm{CH}_{3}$

c: $R_{3}-R_{5}=H_{1}, R_{1}=R_{2}=\mathrm{CH}_{3}$

d: $R_{1}-R_{3}=R_{5}=H, R_{4}=C H_{3}$

$e: R_{2}=R_{3}=R_{5}=H_{1}, R_{1}=R_{4}=C_{3}$<smiles>[R]/C=C/C(=O)O</smiles>

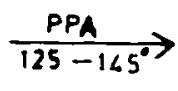

$\underline{4}$

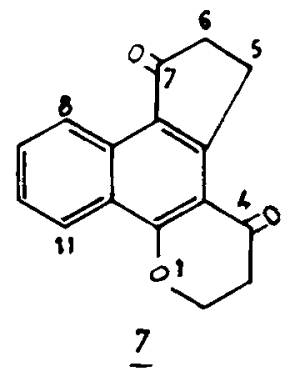<smiles>[R]c1ccc2c(c1[R6])C1=C(CC([Y9])CC1=O)C([R6])C2</smiles>

$\underline{5}$<smiles>O=c1ccoc2c1ccc1ccccc12</smiles>

$\underline{8}$

Scheme 1 .

careful chromatography. Elution with pet. ether $\left(60^{\circ}-80^{\circ}\right)$ : benzene $(1: 1)$ yielded a yellow solid $(60 \%)$. This major product was assigned the structure $5 \mathrm{n}$ on the basis of spectroscopic and analytical data.

The IR spectrum of $5 \mathrm{a}$, the expected product, revealed the presence of an $\alpha$, $\beta$-unsaturated carbonyl group at $1650 \mathrm{~cm}^{-1}$. The ${ }^{1} \mathrm{H}$ NMR spectrum of $\underline{5 \mathrm{a}}$ showed a triplet at $\delta 2.7$ for the methylene protons next to the carbonyl group and a corresponding triplet at $\delta \mathbf{4 . 6 3}$ for the methylene next to the heterocyclic oxygen, indicating the presence of a pyran-4-one structure. Additional proof for this structure was obtained by dehydrogenating $\underline{5 \mathrm{a}}$ with $\mathrm{Pd} / \mathrm{C}$ in refluxing diphenyl ether to $4 \mathrm{H}$ naphtho(1,2-b) pyran-4-one (으). In a separate experiment, $\underline{8}$ was also prepared by a known method reported in the literature (von Strandtmann et al 1972) (mixed melting points and superimposable IR spectra). The mass spectrum $(m / z=328)$ and elemental analysis $\left(\mathrm{C}_{23} \mathrm{H}_{20} \mathrm{O}_{2}\right)$ of the second component (yield $10 \%$ ) suggested that two moles of $3 \mathrm{a}$ had reacted with one mole of acrylic acid. The ${ }^{1} \mathrm{H}$ NMR spectrum was of little help in evaluating the structure because of its complex nature and the close proximity of a number of proton resonances. Its ${ }^{13} \mathrm{C}$ NMR spectrum (proton coupled and decoupled) showed signals corresponding to twenty-three carbon atoms including two carbonyls at $\delta 197.4$ and $\delta 196.7$ and one singlet at $\delta 49.2$ for the spiro carbon. In order to complete the structure elucidation of this compound we relied on the information provided by homonuclear 2D as well as decoupling experiments (figures 1 and 2). 
With the help of the ${ }^{1} \mathrm{H}$ COSY plot of $6 \mathrm{a}$ it was relatively easy to decipher the region between $\delta 6.9$ and 8.2 into two sets (4 protons each) of aromatic protons. The protons of the naphthalene system are deshielded to a larger extent due to the carbonyl function contiguous to the aromatic ring. In the region $\delta$ 1-5-3-5 unambiguous correlation was observed between four protons (magnetically nonequivalent). The molecular model of $\underline{6 a}$ clearly showed such a system $\left(\mathrm{CH}_{2}-\mathrm{CH}_{2}\right)$ as the one present in the ring B. Fortunately, the multiplet pattern at $\delta 3.3$ is readily recognised as a doublet-triplet-doublet because of its symmetry and apparent simplicity. This multiplet can be considered as a pattern of four distorted doublets in which the two inner peaks overlap to give a seven-peak multiplet $\left(J_{\mathrm{avg}}=6.1,13.8,18.02 \mathrm{~Hz}\right)$. Irradiation at $\delta 3.3 \mathrm{ppm}$ resulted in the collapse of the multiplet at $\delta 20$ into a double triplet $(J=10 \cdot 2,3 \cdot 2 \mathrm{~Hz})$. Irradiation at $2.0 \mathrm{ppm}$ resulted in the collapse of the multiplet at $\delta 3.3$ into a double doublet $\left(\mathrm{C}_{4}-\mathrm{H}_{a}, J=18.2,15 \cdot 2 \mathrm{~Hz}\right)$ corresponding to one geminal and one axial-axial interaction. Also, irradiation at $20 \mathrm{ppm}$ resolved the complex multiplet $(2 \mathrm{H})$ at $\delta 2.9-3.05$ into two sets of double doublets. The $d d$ at $\delta 2.93-3.01$ showed $J=18.2 ; 5.0 \mathrm{~Hz}\left(\mathrm{C}_{4}-\mathrm{H}_{e}\right)$ and the $d d$ at $\delta 2.98-3.05$ showed $J=15.1 ; 4.8 \mathrm{~Hz}\left(\mathrm{C}_{3}-\mathrm{H}_{a}\right)$. Irradiation at $2.98 \mathrm{ppm}$ resulted in the collapse of the multiplet at $\delta 3.3$ into a double doublet $(J=15.3 ; 6.7 \mathrm{~Hz})$. Hence the peak at $\delta 2.0$ is due to the equatorial proton at $C_{3}$. The complex multiplet at $\delta 2 \cdot 2-2 \cdot 7$ integrating for eight protons belongs to the phenanthrene nucleus. The regiochemistry of $6 \mathrm{a}$ was thus established.

Mechanistically the formation of 6a could be explained as follows (Rowlands 1985):

(i) 1-tetralone (3a) reacts with its enol form to give intermediate I (Evans and Smith 1954; Metz 1972);

(ii) acylation of intermediate I with acrylic acid to give intermediate II;

(iii) cyclisation of II to give 6 a (scheme 2).

The third compound isolated in $2 \%$ yield showed a strong absorption for the carbonyl group at $1680 \mathrm{~cm}^{-1}$ whereas its mass spectrum gave $M^{+}$at $m / z=252$ with molecular formula $\mathrm{C}_{16} \mathrm{H}_{12} \mathrm{O}_{3}$ which was well supported by its elemental analysis. A comparison with the molecular formula of $5 \mathrm{a}$ indicates the addition of three carbon atoms which could come from acrylic acid moiety. In our earlier work it was observed that the cyclopentanone ring attached to the naphthalene nucleus exhibits IR frequency in the region of $1680 \mathrm{~cm}^{-1}$. Therefore structure $7 \mathrm{a}$ was assigned to this product. Further confirmation was obtained from its ${ }^{1} \mathrm{H}$ NMR spectrum wherein, out of four aromatic protons, the one in the proximity of the carbonyl group was seen at $\delta \mathbf{9 \cdot 2}$. Unambiguous proof for this structure was obtained when the same compound 7 a was isolated from the reaction of $\alpha$-naphthol with acrylic acid in the presence of PPA (mixed melting points and superimposable IR). In order to obtain an insight into the mechanism involved in the formation of this product, compound 5 a was reacted with acrylic acid in the presence of PPA under similar conditions when $\underline{7 a}$ was isolated (mixod melting points and superimposable IR spectra). This clearly indicates that in the reaction $\underline{5 a}$, which is formed first, gets dehydrogenated and further reacts with another molecule of acrylic acid to give $7 \mathrm{a}$. We have also observed that the reaction of 3 a with acrylic acid is highly dependent on the stoichiometry of the reactants. This effect is shown in table $\mathbf{l}$.

We next turned our attention to the reaction of $3 \mathrm{a}$ with crotonic acid in the presence of PPA as described previously in the ratio $1: 2$ respectively. In this case only one 
M1 Data in file $\mathrm{MI}$

Pulse sequence relayh OBSERVE H1

Frequency $299.949 \mathrm{miz}$

Spectral vidth $2271.2 \mathrm{~Hz}$

20 Spectral vidth $2271.2 \mathrm{~Hz}$

Mquisition tim 0.225 s

Relaration tien 1.500 .

Pulse vidth 90.0 dogrees

First polse vidth 90.0 degrees

Teaperature 25.0 dec. C/ 206.1 k

In. repotitions 16

IV. incresents 180

Double precision aquisition

DATA PEOCESSIIIG

Sim boll 0.113 s

T Size 1024

Total aquisition tien ain

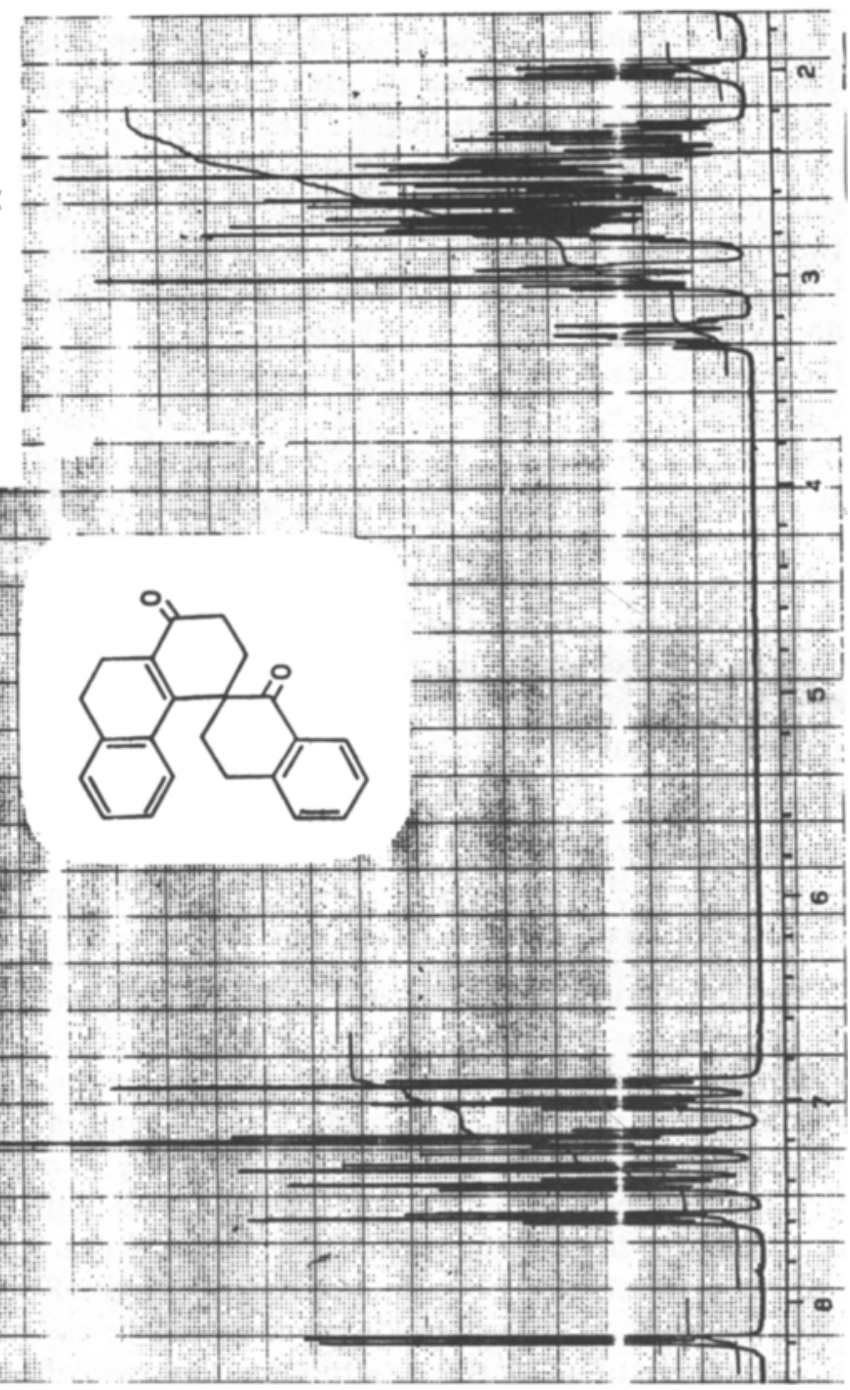

Figure 1a. (Continued facing page.)

compound $5 \mathrm{~b}$ was isolated in $83 \%$ yield. However, when the ratio of the reactants was changed to $2: 1$, compound $\underline{6 b}$ (yield $36 \%$ ), was isolated along with $\underline{5 b}$ (yield $40 \%$ ) after column chromatography.

In order to show the generality of this new method we reacted several methyl and dimethyl 1-tetralones with acrylic as well as crotonic acids in the presence of PPA. In all these cases only the corresponding naphtho(1,2-b) pyrones were isolated in $65-85 \%$ yield. In the case of 4-methyl-1-tetralone ( $3 \mathrm{c})$ and crotonic acid the product $\underline{5 c}$ was 


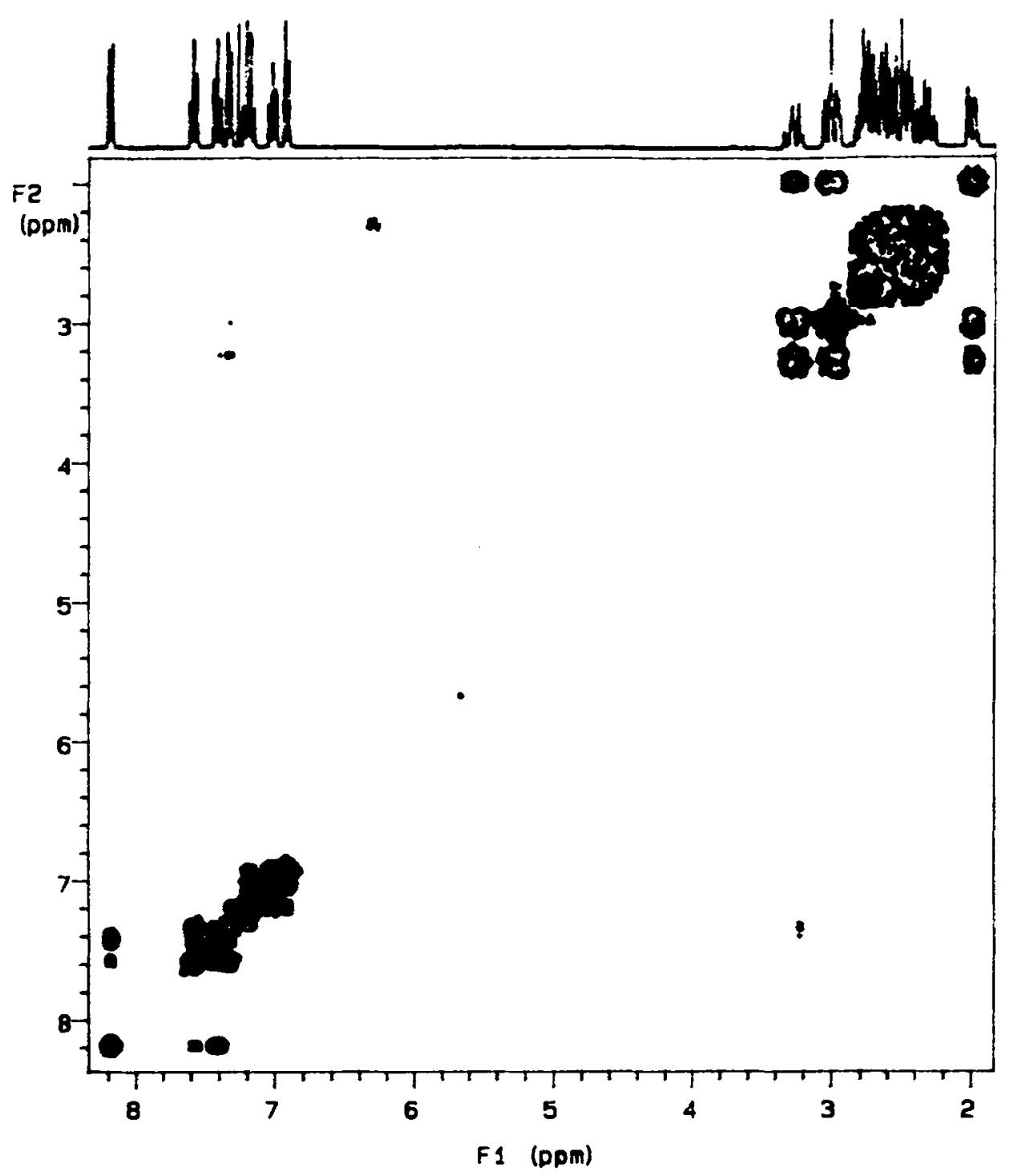

Figure 12. (Caption on p. 115.)

obtained which can exist as two diastereoisomers in the ratio $54: 46$ as evident from its ${ }^{1} \mathrm{H}$ NMR (300 MHz) spectrum.

\section{Experimental section}

All melting points are recorded in open capillaries and are uncorrected. Silica gel (60-120 mesh) was used for chromatography throughout. The homogeneity of compounds was ascertainod by tkc on silica gel-G plates using different solvent systems and spots were developed in an iodine chamber. IR spectra were recorded on a Perkin-Elmer 781 spectrophotometer. UV spectra were recorded on a Shimadzu UV 240 spectrophotometer. 


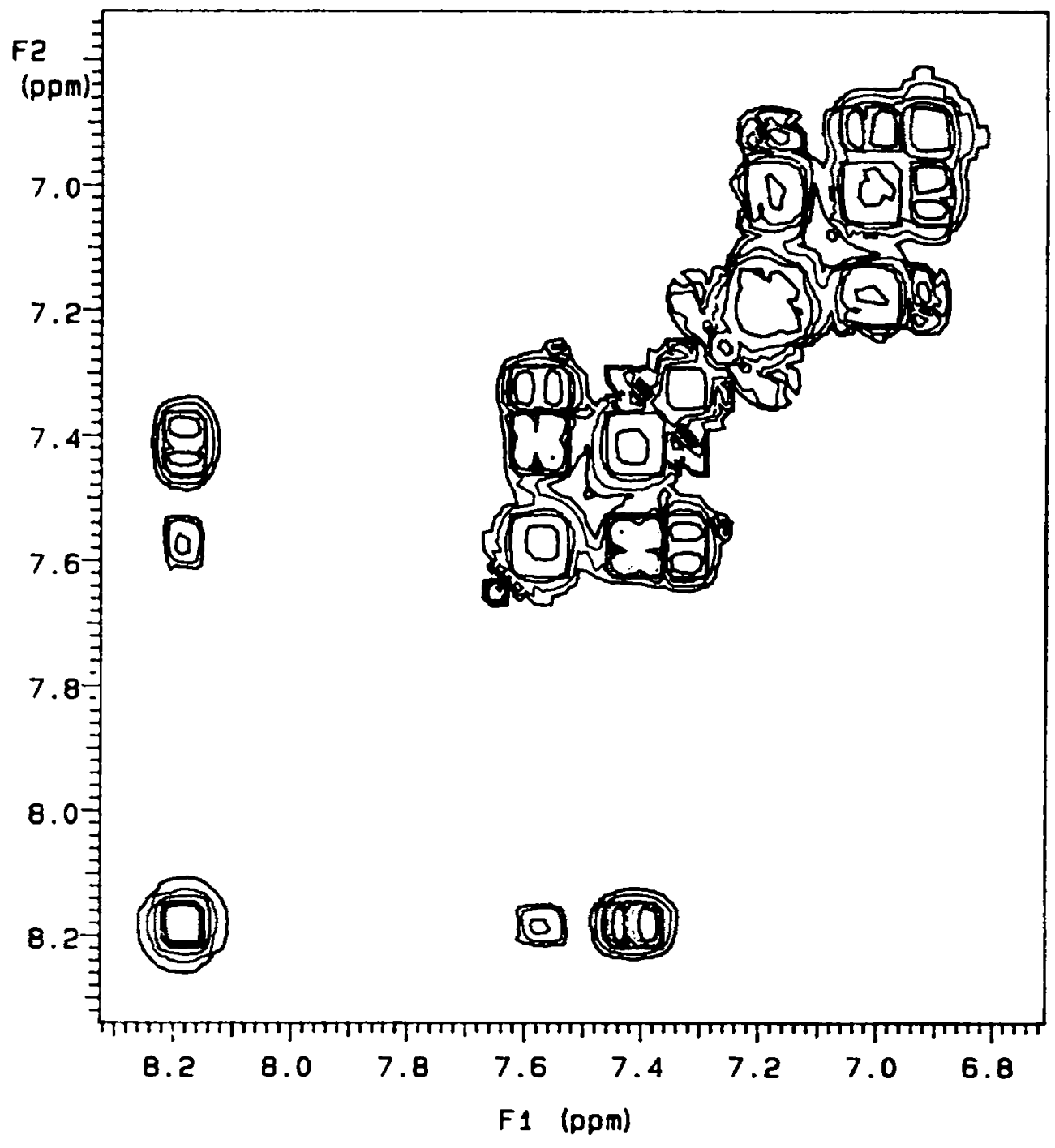

Figure 1b. (Caption on facing page.)

Elemental analysis was done on the Carlo Erba $\mathrm{M} 00-1106$ instrument. ${ }^{1} \mathrm{H}$ and ${ }^{13} \mathrm{C}$ NMR spectra $(300 \mathrm{MHz})$ were recorded at the Regional Sophisticated Instrumentation Centre, Indian Institute of Technology, Bombay on a Varian XL 300. Mass Spectra were obtained using the Finnigan Mat $1020 \mathrm{C}$ automated GC/MS quadrupole mass spectrometer at the National Chemical Laboratories, Pune.

\subsection{Preparation of 1-tetralones}

1-Tetralone ( $\underline{3 \mathrm{a}})$ and 4-methyl-1-tetralone ( $\underline{3 \mathrm{c}})$ were prepared according to the method described in Organic Synthesis (Olson and Bader 1963). Other substituted 1-tetralones (De Barry and Sanders 1933) were prepared by an improved method already described in literature (Eisenbraun et al 1971). 


\section{(C)}

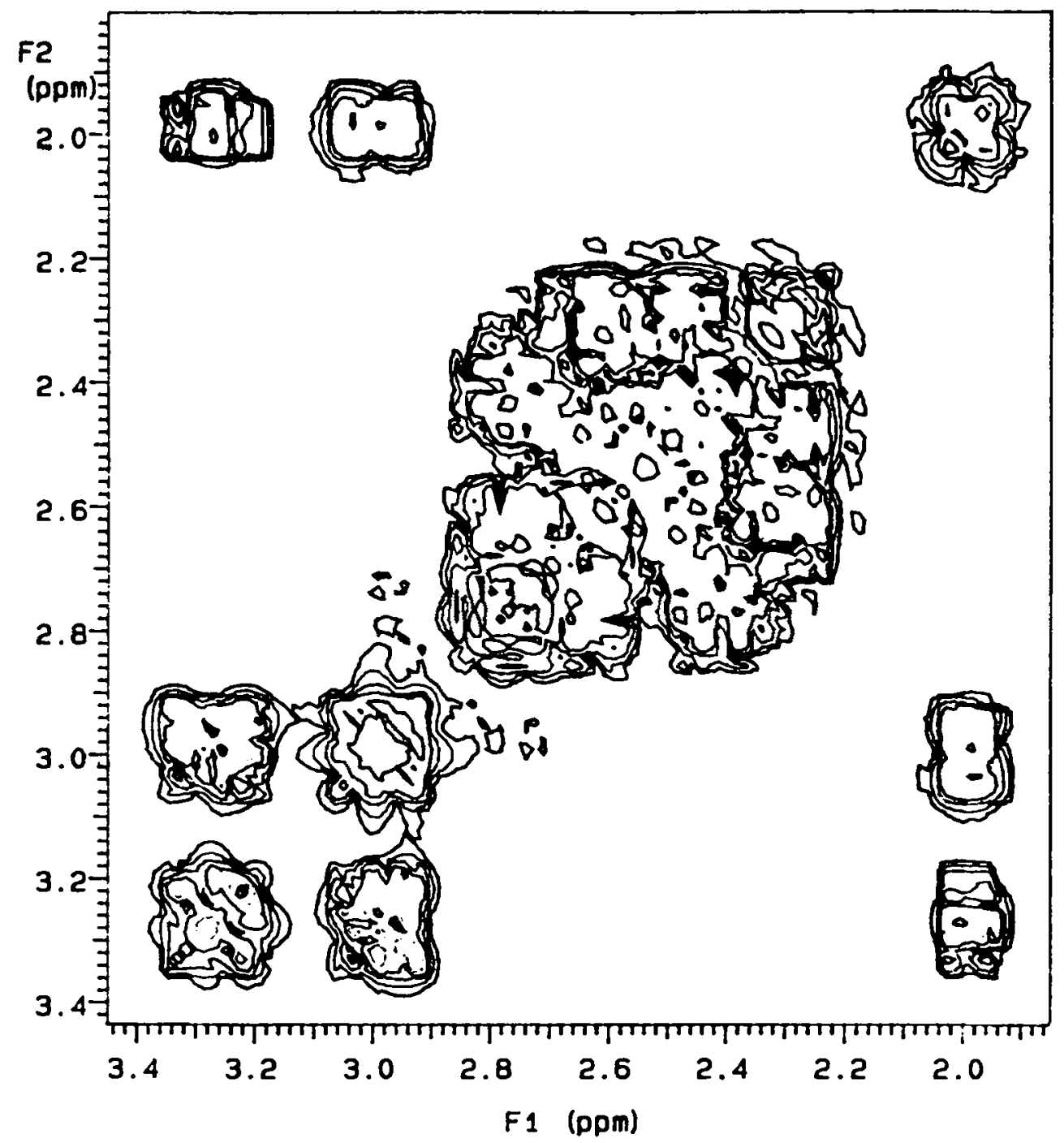

Figure 1. (a) A $300 \mathrm{MHz}{ }^{1} \mathrm{H}$ homonuclear COSY NMR spectrum of $\underline{3 b}$. (b) Expansion of the region $\delta 6.8-8.4 \mathrm{ppm}$. (c) Expansion of region $\delta 1.9-3.4 \mathrm{ppm}$.

3.2 General procedure for the synthesis of 2,3,5.6-tetrahydro-4H-naphtho(1,2-b) pyran-4-ones

To freshly prepared polyphosphoric acid (PPA) [40 g, prepared from phosphorus pentoxide $(20 \mathrm{~g})$ and phosphoric acid $(>98 \%, 10 \mathrm{ml})$ heated to $100^{\circ} \mathrm{C}$ for $\left.0.5 \mathrm{~h}\right]$ preheated to $125^{\circ} \mathrm{C}$, were added the respective 1-tetralone $(\approx 2 \mathrm{~g}, 0.015 \mathrm{~mol})$ and acrylic acid $(2.2 \mathrm{~g}, 0.03 \mathrm{~mol})$. The reaction mixture was maintained at this temperature for 4.5 to $5.0 \mathrm{~h}$ with mechanical stirring. It was then cooled, quenched with ice water and left overnight. The solid obtained was separated by filteration. The residue as well as the 
filtrate were extracted separately with chloroform. The combined chloroform extracts were washed thoroughly with sodium hydroxide $(10 \%)$, water and dried $\left(\mathrm{MgSO}_{4}\right)$. Evaporation of the solvent left an oily residue which was chromatographed on silica gel. Elution with appropriate solvents yielded the respective products.

(a)

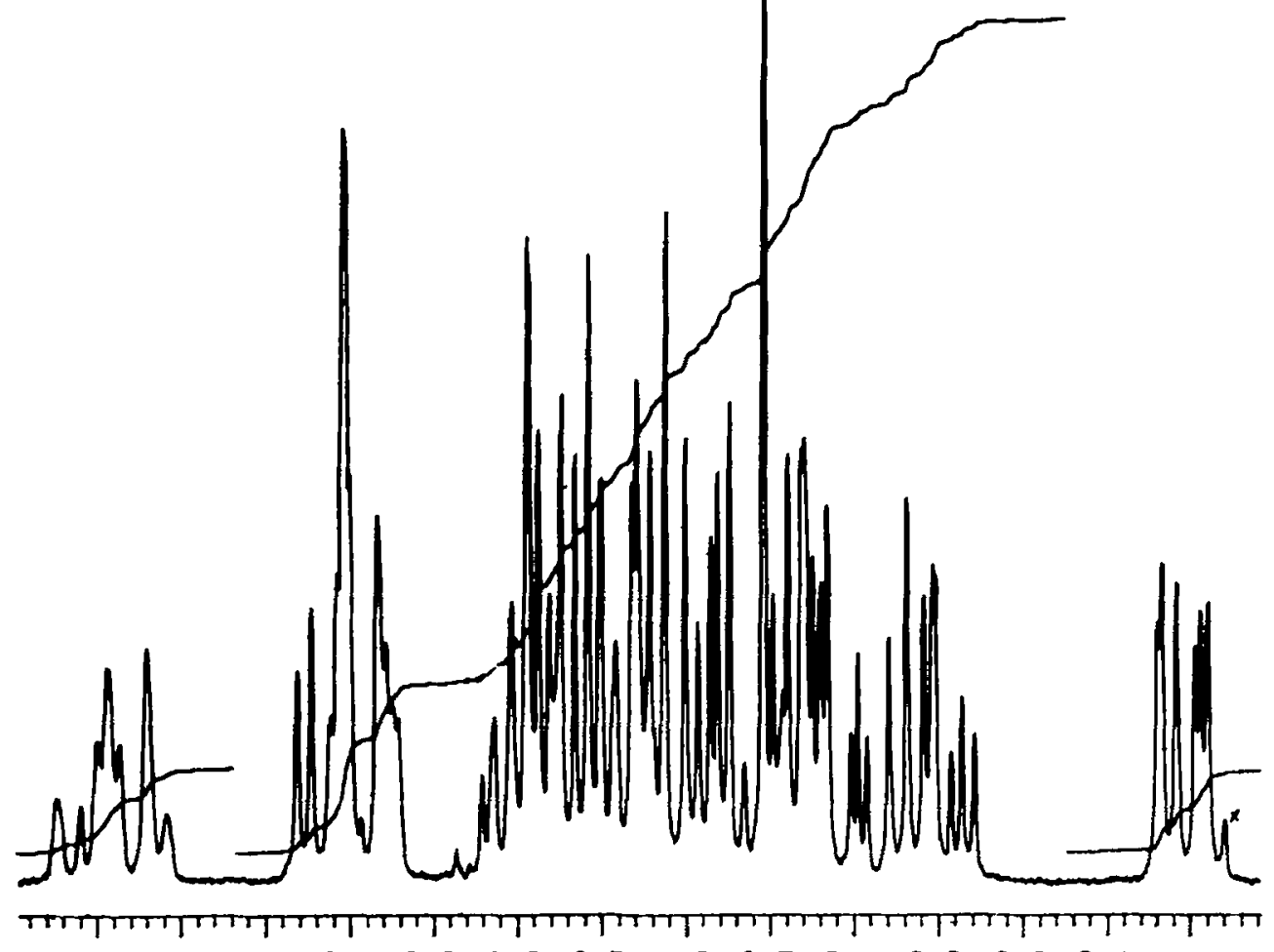

$\begin{array}{llllllllllllll}3.3 & 3.2 & 3.1 & 3.0 & 2.9 & 2.8 & 2.7 & 2.6 & 2.5 & 2.4 & 2.3 & 2.2 & 2.1 & \text { ppm }\end{array}$

(b)

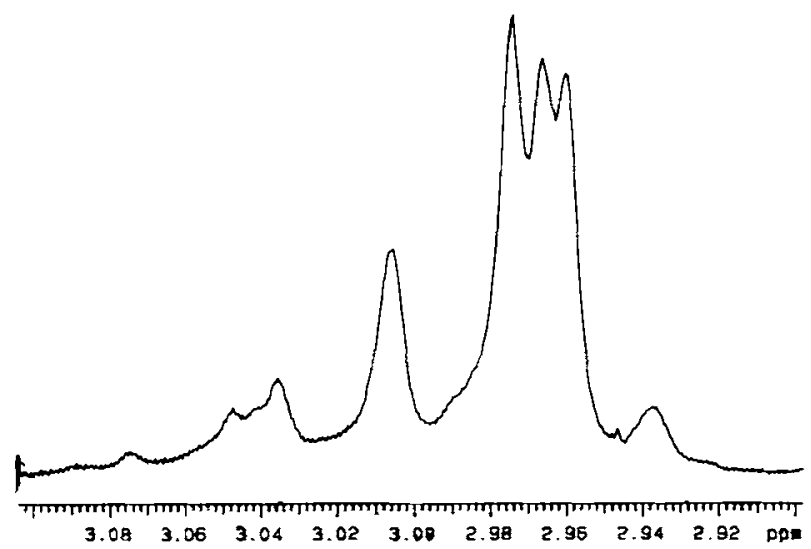

Figure 2. (a and b) (Caption on facing page.) 

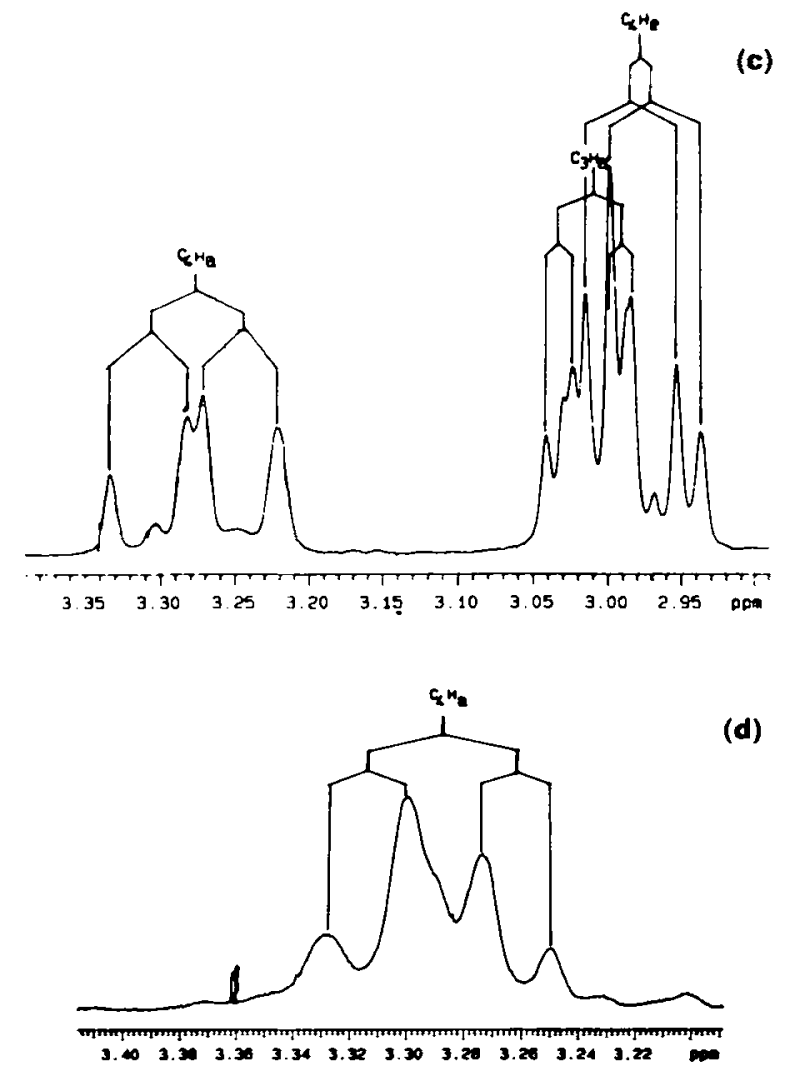

(d)

Figure 2. $300 \mathrm{MHz}^{1} \mathrm{H}$ NMR spectrum of $3 \mathrm{~b}$, region $\delta 1.9-3.4(\mathrm{a})$, decoupled at $\delta 3.3 \mathrm{ppm}(\mathrm{b}), \delta 2.0 \mathrm{ppm}(\mathrm{c})$, and $\delta 2.98 \mathrm{ppm}(\mathrm{d})$.

e:

$R_{1}-R_{5}=H: 2,3,5,6-$ Tetrahydro-4H-naphtho(1,2,-b)pyran-4-one (5a): Pale yellow crystals from benzene: pet. ether $(60-80)$, m.p., $60^{\circ} \mathrm{C}$. UV $\lambda^{\mathrm{MeOH}} \mathrm{nm}(\log \varepsilon)$ : $210(4-0)$, 232(4.3), 325(4.4). IR(KBr) $\left(v, \mathrm{~cm}^{-1}\right)$ : $1650(\mathrm{C}=0), 1620,1595(\mathrm{Ar}) .{ }^{1} \mathrm{H} \mathrm{NMR}(300 \mathrm{MHz}$, $\left.\mathrm{CDCl}_{3}\right) \delta: 2 \cdot 62\left(t, J=8.23 \mathrm{~Hz}, 2 \mathrm{H}, \mathrm{CH}_{2}-5\right), 2 \cdot 7\left(t, J=6.8 \mathrm{~Hz}, 2 \mathrm{H}, \mathrm{CH}_{2}-3\right), 2 \cdot 82(t$, $\left.J=8.23 \mathrm{~Hz}, 2 \mathrm{H}, \mathrm{CH}_{2}-6\right), 4 \cdot 63\left(t, J=6.8 \mathrm{~Hz}, 2 \mathrm{H}, \mathrm{CH}_{2}-2\right), 7 \cdot 22(d d, J=7 \cdot 3 \mathrm{~Hz}, 1 \mathrm{H}$, $\mathrm{CH}-7), 7.28(d t, J=7.5,1.46 \mathrm{~Hz}, 1 \mathrm{H}, \mathrm{CH}-8), 7.36(d t, J=7.3,1.46 \mathrm{~Hz}, 1 \mathrm{H}, \mathrm{CH}-9)$, $7 \cdot 69\left(d d, J=7 \cdot 5,1 \cdot 3 \mathrm{~Hz}, 1 \mathrm{H}, \mathrm{CH}-10\right.$ ). Analysis - Calculated for $\mathrm{C}_{13} \mathrm{H}_{12} \mathrm{O}_{2}: \mathrm{C}, 78.0$; H, 6.0\%. Found: C, $77.9 ; \mathrm{H}, 6 \cdot 2 \%$. MS: $(\mathrm{m} / z, 70 \mathrm{ev}): 200\left(M^{+}\right), 172,156,144,118,115,90$.

2,4-Dinitrophenylhydrazone derivative: Orange red crystals from benzene, m.p. 243$44^{\circ} \mathrm{C}$. Analysis - Calculated for $\mathrm{C}_{19} \mathrm{H}_{15} \mathrm{~N}_{4} \mathrm{O}_{5}: \mathrm{N}, 14 \cdot 8 \%$. Found: $\mathrm{N}, 15 \cdot 0 \%$.

Semicarbazone derivative: Pale yellow needles from benzene: pet. ether $(60-80)$, m.p. 231-32 ${ }^{\circ} \mathrm{C}$. Analysis - Calculated for $\mathrm{C}_{14} \mathrm{H}_{15} \mathrm{~N}_{3} \mathrm{O}_{2}: \mathrm{N}, 16 \cdot 3 \%$. Found: $\mathrm{N}, 16 \cdot 15 \%$.

$R_{1}-R_{5}=H: 2^{\prime}, 3,3^{\prime}, 4^{\prime}, 9^{\prime}, 10^{\prime}$-Hexahydrospiro [naphthalene-2,4'-phenanthrene]-1,1'dione $(\underline{6 a})$ : Crystallised from chloroform: ethanol as colourless prisms, m.p. $180-81^{\circ} \mathrm{C}$. UV $\lambda^{\mathrm{MeOH}} \mathrm{nm}(\log \varepsilon): 212(4 \cdot 2), 237(4 \cdot 1), 252(4 \cdot 2), 298(4 \cdot 2) . \operatorname{IR}(\mathrm{KBr})\left(v, \mathrm{~cm}^{-1}\right): 1695,1680$ $(\mathrm{C}=\mathrm{O}) ; 1620,1580(\mathrm{Ar}) .{ }^{1} \mathrm{H}$ NMR $\left(300 \mathrm{MHz}, \mathrm{CDCl}_{3}\right) \delta: 2 \cdot 0(m, J=10 \cdot 2,6 \cdot 4,3 \cdot 2$ 

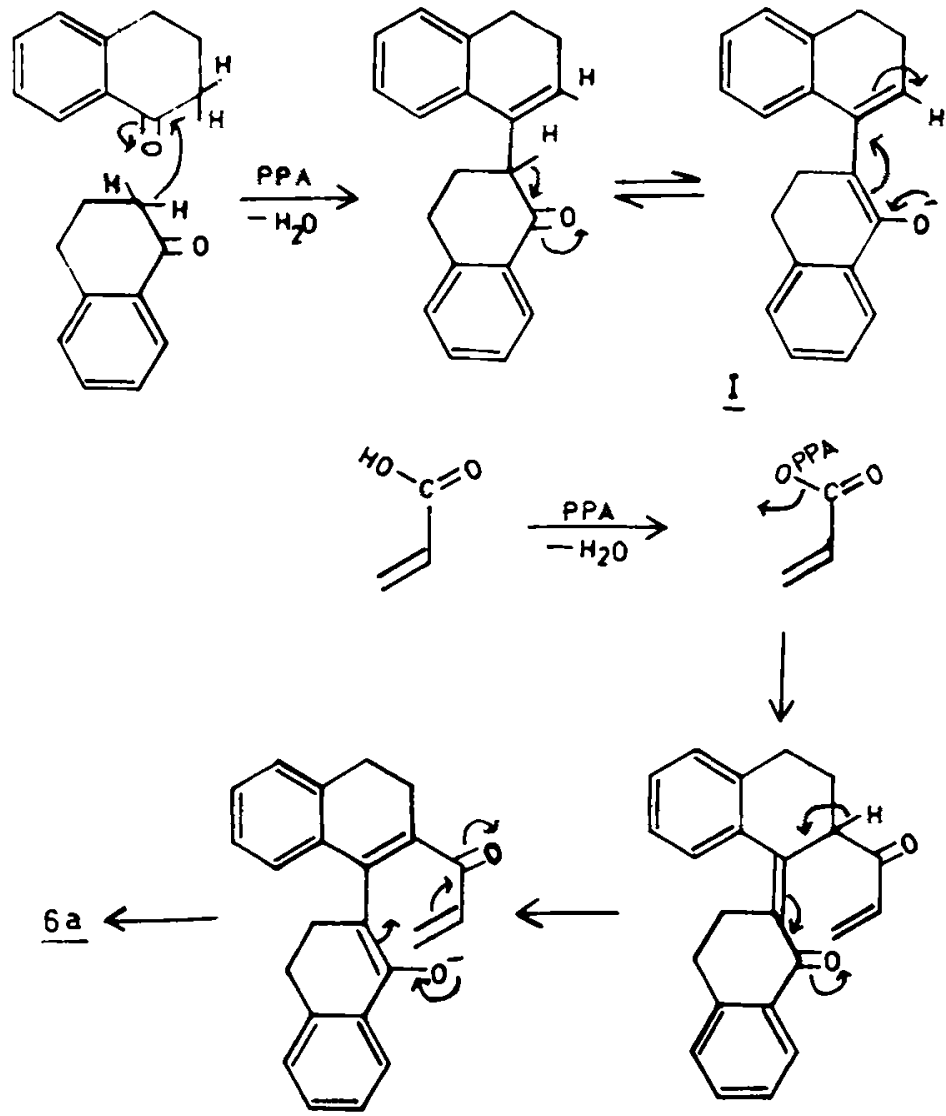

II

Scheme 2.

$\left.\mathrm{Hz}, 1 \mathrm{H}, \mathrm{CH}_{a}-3\right), 2 \cdot 24-2 \cdot 9\left(m, 8 \mathrm{H}, \mathrm{CH}_{2}-2^{\prime}, 3^{\prime}, 9^{\prime}, \mathbf{1 0}^{\prime}\right), 2 \cdot 9-3 \cdot 01(\mathrm{br} m, J=18 \cdot 2,4 \cdot 9,3 \cdot 2$ $\left.\mathrm{Hz}, 1 \mathrm{H}, \mathrm{CH}_{e}-4\right), 2 \cdot 98-3 \cdot 05\left(\mathrm{brm}, J=15 \cdot 1,10 \cdot 1,4 \cdot 9 \mathrm{~Hz}, 1 \mathrm{H}, \mathrm{CH}_{a}-3\right), \quad 3 \cdot 29(d d d, J=$ $\left.18 \cdot 2,15 \cdot 2,6 \cdot 4 \mathrm{~Hz}, 1 \mathrm{H}, \mathrm{CH}_{a}-4\right), 6.92(d, J=7 \cdot 7 \mathrm{~Hz}, \mathrm{CH}-8), 7 \cdot 02(d t, J=7.9,2 \cdot 0 \mathrm{~Hz}, 1 \mathrm{H}$, $\mathrm{CH}-6), \quad 7 \cdot 14-7 \cdot 25\left(\mathrm{~m}, 2 \mathrm{H}, \mathrm{CH}-5^{\prime}, 7^{\prime}\right), \quad 7 \cdot 33(d, 1 \mathrm{H}, \mathrm{CH}-5), \quad 7 \cdot 43(t, J=7 \cdot 7 \mathrm{~Hz}, 1 \mathrm{H}$, $\mathrm{CH}-7), \quad 7 \cdot 58(d t, J=7 \cdot 8,1 \cdot 5 \mathrm{~Hz}, 1 \mathrm{H}, \mathrm{CH}-6), \quad 8 \cdot 2(d d, J=7 \cdot 9,1.3 \mathrm{~Hz}, 1 \mathrm{H}, \mathrm{CH}-8)$. ${ }^{13} \mathrm{CNMR} \quad\left(300 \mathrm{MHz}_{2} \mathrm{CDCl}_{3}\right)$ : 197.42(s), 196.75(s), 151.9(s), 142.1(s), 139.42(s), $136.48(s), 133 \cdot 74(d), 131 \cdot 32(s), 130.68(s), 128 \cdot 77(d), 128 \cdot 42(d), 128 \cdot 15(d), 127 \cdot 89(d)$, 127.04(d), 126.92(d), 125.44(d), 49.19(s, C 2-4'), 33.31(t), 31.84(t), 29.28(t), 27.73(t), 24.85(t), 20-54(t). Analysis - Calculated for $\mathrm{C}_{23} \mathrm{H}_{20} \mathrm{O}_{2}: \mathrm{C}, 84 \cdot 14 ; \mathrm{H}, 6.09 \%$. Found: C, 84-0; H, 5.98\%. MS (m/z, 70 ev): 328 $\left(M^{+}\right), 224,196,181,168,139$.

2,4-Dinitrophenylhydrazone derivative: Orange crystals from chloroform:benzene, m.p. $254-55^{\circ} \mathrm{C}$. Analysis - Calculated for $\mathrm{C}_{29} \mathrm{H}_{24} \mathrm{~N}_{4} \mathrm{O}_{5}: \mathrm{N}, 11 \cdot 02 \%$. Found: $\mathrm{N}, 11 \cdot 2 \%$.

$R_{1}-R_{5}=H: 2,3,5,6-$ Tetrahydrocyclopenta $\left[3^{\prime}, 2^{\prime}-3,4\right]$ naphtho(1,2-b)-pyran-4,7-dione (7a): Crystallised from chloroform:pet. ether $(60-80)$ as colourless needles, m.p. 
Table 1. Reaction of 1-tetralone (3) with acrylic acid.

\begin{tabular}{|c|c|c|c|c|}
\hline $\begin{array}{l}\text { 1-Tetralone } \\
\text { (moles) }\end{array}$ & $\begin{array}{l}\text { Acrylic acid } \\
\text { (moles) }\end{array}$ & $\begin{array}{c}\text { Temperature } \\
\left({ }^{\circ} \mathrm{C}\right)\end{array}$ & $\begin{array}{c}\text { Eluting solvent } \\
\quad X: Y: Z\end{array}$ & $\begin{array}{l}\text { Products } \\
\text { (\% yield) }\end{array}$ \\
\hline 1 & 2 & 125 & $\begin{array}{l}1: 1:- \\
-: 1:- \\
-: 8: 2\end{array}$ & $\begin{array}{l}5 a(60) \\
6 a(10) \\
7 a(02)\end{array}$ \\
\hline 2 & 1 & $135-140$ & $\begin{array}{l}1: 1:- \\
-: 1:- \\
-: 8: 2\end{array}$ & $\begin{array}{l}5 \mathrm{a}(08) \\
6 \mathrm{a}(65) \\
7 \mathrm{a}(\mathrm{nil})\end{array}$ \\
\hline 1 & 2 & $140-145$ & $\begin{array}{r}1: 1:- \\
-: 1:- \\
-:: 8: 2\end{array}$ & $\begin{array}{l}5 a(50) \\
6 a(20) \\
7 a(10)\end{array}$ \\
\hline
\end{tabular}

$X=$ pet. ether $\left(60^{\circ}-80^{\circ}\right), Y=$ benzene, $Z=$ chloroform.

276-77 ${ }^{\circ} \mathrm{C}$. UV $\lambda^{\mathrm{MeOH}} \mathrm{nm}(\log \varepsilon): 277(4 \cdot 9), 278(4 \cdot 9), 290(4 \cdot 7), 305(4-8), 314(4 \cdot 8), 354(4 \cdot 6)$, 369(4.6). IR(KBr) $\left(v, \mathrm{~cm}^{-1}\right): 1680(s, \mathrm{C}=\mathrm{O}), 1620,1548(\mathrm{Ar}) .{ }^{1} \mathrm{H}$ NMR (300 MHz, $\left.\mathrm{CDCl}_{3}\right)$ $\delta: 2.75\left(m, 2 \mathrm{H}, \mathrm{CH}_{2}-6\right), 2.96\left(t, J=6.6 \mathrm{~Hz}, 2 \mathrm{H}, \mathrm{CH}_{2}-3\right), 3.53\left(\mathrm{~m}, 2 \mathrm{H}, \mathrm{CH}_{2}-5\right), 4.85(t$, $\left.J=6.6 \mathrm{~Hz}, 2 \mathrm{H}, \mathrm{CH}_{2}-2\right), 7.59(d d d, J=7.02,8.39,1.37 \mathrm{~Hz}, 1 \mathrm{H}, \mathrm{CH}-10), 7.79$ (ddd, $J=8.24,7.02,1.37 \mathrm{~Hz}, 1 \mathrm{H}, \mathrm{CH}-9$ ), $8.32(d d d, J=8 \cdot 39,1 \cdot 37,0.765 \mathrm{~Hz}, 1 \mathrm{H}, \mathrm{CH}-8$ ), 9.2(d, $J=8 \cdot 24 \mathrm{~Hz}, 1 \mathrm{H}, \mathrm{CH}-11)$. Analysis - Calculated for $\mathrm{C}_{16} \mathrm{H}_{12} \mathrm{O}_{3}: \mathrm{C}, 76.19$; H, 4.76\%. Found: C, 76.32; H,4.5\%. MS: $(m / z, 70 \mathrm{ev}): 252\left(M^{+}\right), 224,196,181,168,139$.

2,4-Dinitrophenylhydrazone derivative: Bright red crystals from dioxane:chloroform, m.p. $332-33^{\circ} \mathrm{C}$ with decomposition. Analysis-Calculated for $\mathrm{C}_{22} \mathrm{H}_{15} \mathrm{~N}_{4} \mathrm{O}_{6}$ : N, 12.99\%. Found: N, 12.85\%.

$R_{2}-R_{5}=H, R_{1}=C_{3}: 2-M e t h y l-2,3,5,6-t e t r a h y d r o-4 H$-naphtho(1,2-b)-pyran-4-one ( $\$ b$ ): Pale yellow crystals from benzene:pet. ether $(60-80)$, m.p. $73-74^{\circ} \mathrm{C}$. IR(KBr) $\left(v, \mathrm{~cm}^{-1}\right): 1650(\mathrm{C}=\mathrm{O}), 1610,1595(\mathrm{Ar}) .{ }^{1} \mathrm{H} \mathrm{NMR}\left(80 \mathrm{MHz}, \mathrm{CDCl}_{3}\right) \delta: 1 \cdot 59(d, J=6 \cdot 32 \mathrm{~Hz}$, $1 \mathrm{H}, \mathrm{CH}-2), 2 \cdot 5-2 \cdot 8\left(m, 6 \mathrm{H}, \mathrm{CH}_{2}-3,5,6\right), 4 \cdot 4-4 \cdot 9(m, 1 \mathrm{H}, \mathrm{CH}-2), 7 \cdot 23-7 \cdot 34(m, 3 \mathrm{H}$, $\mathrm{CH}-7,8,9), 7 \cdot 7(d, 1 \mathrm{H}, \mathrm{CH}-10)$. Analysis - Calculated for $\mathrm{C}_{14} \mathrm{H}_{14} \mathrm{O}_{2}: \mathrm{C}, 78 \cdot 5$; H, 6.54\%. Found: C, 78.3; H, 6.41\%.

2,4-Dinitrophenylhydrazone derivative: Red crystals from benzene, m.p. $252^{\circ} \mathrm{C}$.

$R_{2}-R_{5}=H, R_{1}=\mathrm{CH}_{3}: 2^{\prime}, 3,3^{\prime}, 4,9^{\prime}, 10^{\prime}$-Hexahydro-3'-methylspiro[naphthalene-2,4'phenanthrene]-1, $1^{\prime}$-dione $(\underline{6 b})$ : Shining prisms from chloroform:ethanol, m.p. $228^{\circ} \mathrm{C}$. $\operatorname{IR}(\mathrm{KBr})\left(v, \mathrm{~cm}^{-1}\right): 1660(\mathrm{sbr}, \mathrm{C}=\mathrm{O}), 1610,1560(\mathrm{Ar}) .{ }^{1} \mathrm{H} \mathrm{NMR}\left(300 \mathrm{MHz}, \mathrm{CDCl}_{3}\right) \delta$ : $1.25\left(d, J=6.87 \mathrm{~Hz}, 3 \mathrm{H}, \mathrm{CH}_{3}-3^{\prime}\right), 2.0\left(m, 1 \mathrm{H}, \mathrm{CH}_{e}-3\right), 2 \cdot 1-2.9\left(m, 6 \mathrm{H}, \mathrm{CH}_{2}-2^{\prime}, 9^{\prime}, 10^{\prime}\right.$ \& $\left.\mathrm{CH}-3^{\prime}\right), 3 \cdot 0\left(m, 2 \mathrm{H}, \mathrm{CH}_{e}-4 \mathrm{CH}_{a}-3\right), 3 \cdot 2\left(m, 1 \mathrm{H}, \mathrm{CH}_{a}-4\right), 6 \cdot 9(d, J=7.8 \mathrm{~Hz}, 1 \mathrm{H}$, $\left.\mathrm{CH}-8^{\prime}\right), 7 \cdot 03\left(t, 1 \mathrm{H}, \mathrm{CH}-6^{\prime}\right), 7 \cdot 12-7,24\left(m, 2 \mathrm{H}, \mathrm{CH}-5^{\prime}, 7^{\prime}\right), 7 \cdot 32(d, 1 \mathrm{H}, \mathrm{CH}-5), 7 \cdot 41$ $(t, 1 \mathrm{H}, \mathrm{CH}-7), 7 \cdot 57(d t, 1 \mathrm{H}, \mathrm{CH}-6), 8 \cdot 22(d, 1 \mathrm{H}, \mathrm{CH}-8)$, Analysis - Calculated for $\mathrm{C}_{24} \mathrm{H}_{22} \mathrm{O}_{2}: \mathrm{C}, 84 \cdot 2 ; \mathrm{H}, 6 \cdot 43 \%$. Found: $\mathrm{C}, 84 \cdot 3 ; \mathrm{H}, 6 \cdot 3 \%$.

$R_{3}-R_{5}=H, R_{1}=R_{2}=C_{3}: 2,6$-Dimethyl-2,3,5,6-tetrahydro-4H-naphtho(1,2-b) pyran-4-one $(\underline{5 c})$ : 4-Methyl-1-tetralone $(2 \mathrm{~g}, 0.013 \mathrm{~mol})$ reacted with crotonic acid $(1.9 \mathrm{~g}, 0.028 \mathrm{~mol})$. Elution of the chromatogram with pet. ether $(60-80)$ : benzene $(60: 40)$ gave a pale yellow oil; yield $86 \%$. On keeping the oil solidified and crystallised from 
benzene:pet. ether $(60-80)$ into colourless needles, m.p. $55-56^{\circ} \mathrm{C}$. $\operatorname{IR}(\mathrm{K} \mathrm{Br})\left(v, \mathrm{~cm}^{-1}\right)$ : $1660(\mathrm{C}=\mathrm{O}), 1610,1595(\mathrm{Ar}) .{ }^{1} \mathrm{H}$ NMR $\left.(300 \mathrm{MHz}), \mathrm{CDCl}_{3}\right) \delta: 1 \cdot 23-1 \cdot 26(d, J=9 \cdot 46 \mathrm{~Hz}$, $\left.3 \mathrm{H}, \mathrm{CH}_{3}-6\right), 1 \cdot 20-1 \cdot 24\left(d, J=9 \cdot 3 \mathrm{~Hz}, 3 \mathrm{H}, \mathrm{CH}_{3}-6\right), 1 \cdot 549-1 \cdot 569(d, J=6.26 \mathrm{~Hz}, 3 \mathrm{H}$, $\left.\mathrm{CH}_{3}-2\right), 1 \cdot 546-1 \cdot 567\left(d, J=6.4 \mathrm{~Hz}, 3 \mathrm{H}, \mathrm{C}_{3}-2\right), 2 \cdot 38-2 \cdot 73\left(m, 4 \mathrm{H}, 2 \mathrm{CH}_{2}-3,5\right), 4.58-$ $4 \cdot 68(m, 1 \mathrm{H}, 2 \mathrm{CH}-2), 7 \cdot 2-7 \cdot 32(m, 2 \mathrm{H}, 2 \mathrm{CH}-7,9), 7 \cdot 34-7 \cdot 42(t, 1 \mathrm{H}, 2 \mathrm{CH}-8), 7 \cdot 72-$ $7 \cdot 76(d d, J=7 \cdot 6,1 \cdot 4 \mathrm{~Hz}, 1 \mathrm{H}, 2 \mathrm{CH}-10)$. Analysis - Calculated for $\mathrm{C}_{15} \mathrm{H}_{16} \mathrm{O}_{2}: \mathrm{C}, 78.95$; H, $7.02 \%$. Found: C, $78.8 ; \mathrm{H}, 6.9 \%$.

$R_{1}-R_{3}=R_{5}=H, R_{4}=C_{3}: 9-M e t h y l-2,3,5,6$-tetrahydro-4H-naphtho(1,2-b)pyran4-one ( $\underline{5 d})$ : 7-Methyl-1-tetralone (Eisenbraun et al 1971) $(2.0 \mathrm{~g}, 0-013 \mathrm{~mol}$ ) reacted with acrylic acid $(1.9 \mathrm{~g}, 0.028 \mathrm{~mol})$ in PPA $(20 \mathrm{~g})$. Elution of the chromatogram with pet. ether $(60-80)$ : benzene (50:50) afforded the naphthopyrone as an oil which was purified by bulb-to-bulb distillation $\left(170^{\circ} \mathrm{C} / 0.8 \mathrm{~mm}\right)$; yield $64 \%$. IR(neat) $\left(v, \mathrm{~cm}^{-1}\right): 1660(\mathrm{C}=\mathrm{O})$, $1610,1565(\mathrm{Ar}) .{ }^{1} \mathrm{H}$ NMR $\left(80 \mathrm{MHz}, \mathrm{CDCl}_{3}\right) \delta: 2 \cdot 35\left(\mathrm{~s}, 3 \mathrm{H}, \mathrm{CH}_{3}-9\right), 2 \cdot 4-2 \cdot 9(\mathrm{~m}, 6 \mathrm{H}$, $\left.\mathrm{CH}_{2}-3,5,6\right), 4.6\left(t, J=6.4 \mathrm{~Hz}, 2 \mathrm{H}, \mathrm{CH}_{2}-2\right), 7 \cdot 1(d, J=7.8 \mathrm{~Hz}, 1 \mathrm{H}, \mathrm{CH}-7), 7 \cdot 3(d$, $J=7.8 \mathrm{~Hz}, 1 \mathrm{H}, \mathrm{CH}-8), 7.53(s, 1 \mathrm{H}, \mathrm{CH}-10)$. Analysis - Calculated for $\mathrm{C}_{14} \mathrm{H}_{14} \mathrm{O}_{2}$ : C, $78 \cdot 5 ; \mathrm{H}, 6.5 \%$. Found: $\mathrm{C}, 78 \cdot 63 ; \mathrm{H}, 6 \cdot 3 \%$.

2,4-Dinitrophenylhydrazone derivative: Orange-red crystals from benzene, m.p. 218$19^{\circ} \mathrm{C}$. Analysis - Calculated for $\mathrm{C}_{20} \mathrm{H}_{15} \mathrm{~N}_{4} \mathrm{O}_{5}: \mathrm{N}, 14 \cdot 5 \%$. Found: $\mathrm{N}, 14 \cdot 3 \%$.

$R_{2}=R_{3}=R_{5}=H ， \quad R_{1}=R_{4}=C_{3}: \quad 2,9$-Dimethyl-2,3,5,6-tetrahydro-4H-naphtho(1,2-b)pyran-4-one (5e): 7-Methyl-1-tetralone (Eisenbraun et al 1971) $2 \mathrm{~g}, 0.013 \mathrm{~mol}$ ) reacted with crotonic acid $(2.4 \mathrm{~g}, 0.028 \mathrm{~mol})$ in PPA $(20 \mathrm{~g})$. Elution of the chromatogram with pet. ether (60-80): benzene (60:40) afforded the naphthopyrone as a red oil which was purified by bulb-to-bulb distillation $\left(188^{\circ} \mathrm{C} / 2 \mathrm{~mm}\right)$; yield $63 \%$. IR (neat) $\left(v, \mathrm{~cm}^{-1}\right)$ : $1660(\mathrm{C}=\mathrm{O}) ; 1610,1565$ (Ar). ${ }^{1} \mathrm{H}$ NMR (80 MHz, $\left.\mathrm{CDCl}_{3}\right) \delta: 1 \cdot 5\left(d, J=6.2 \mathrm{~Hz}, 3 \mathrm{H}, \mathrm{CH}_{3}-2\right)$, $2 \cdot 34\left(s, 3 \mathrm{H}, \mathrm{CH}_{3}-9\right), 2 \cdot 5-2 \cdot 8\left(m, 6 \mathrm{H}, \mathrm{CH}_{2}-3,5,6\right), 4 \cdot 6(m, 1 \mathrm{H}, \mathrm{CH}-2), 7 \cdot 0(d, J=7 \cdot 8 \mathrm{~Hz}$, $1 \mathrm{H}, \mathrm{CH}-7), 7.2(d, J=7.8 \mathrm{~Hz}, \mathrm{H}, \mathrm{CH}-8), 7.45(\mathrm{~s}, 1 \mathrm{H}, \mathrm{CH}-10)$. Analysis - Calculated for $\mathrm{C}_{15} \mathrm{H}_{16} \mathrm{O}_{2}: \mathrm{C}, 78 \cdot 9 ; \mathrm{H}, 7 \cdot 0 \%$. Found: $\mathrm{C}, 78 \cdot 4 ; \mathrm{H}, 7 \cdot 4 \%$.

2,4-Dinitraphenylhydrazone derivative: Orange red crystals from benzene, m.p. 210$11^{\circ} \mathrm{C}$. Analysis - Calculated for $\mathrm{C}_{21} \mathrm{H}_{20} \mathrm{~N}_{4} \mathrm{O}_{5}: \mathrm{N}, 13.7 \%$. Found: N, $13.3 \%$.

$R_{1}=R_{2}=R_{4}=H, \quad R_{3}=R_{5}=C_{3}:$ 7,10-Dimethyl-2,3,5,6-tetrahydro-4H-naphtho(1,2-b)pyran-4-one (5f): 5,8-Dimethyl-1-tetralone (Eisenbraun et al 1971) (2g, $0.012 \mathrm{~mol})$ reacted with acrylic acid $(1.8 \mathrm{~g}, 0.024 \mathrm{~mol})$ in PPA $(20 \mathrm{~g})$. Elution of the chromatogram with pet. ether:benzene $(50: 50)$ afforded the naphthopyrone as a pale yellow oil which was purified by bulb-to-bulb distillation $\left(192^{\circ} \mathrm{C} / 1 \mathrm{~mm}\right)$; yield $78 \%$. IR (neat) $\left(v, \mathrm{~cm}^{-1}\right): 1690(\mathrm{C}=\mathrm{O}), 1630,1612(\mathrm{Ar}) .{ }^{1} \mathrm{H} \mathrm{NMR}\left(80 \mathrm{MHz}^{\mathrm{CDCl}} \mathrm{CDC}_{3}\right) \delta: 2 \cdot 16(\mathrm{~s}, 3 \mathrm{H}$, $\left.\mathrm{CH}_{3}-7\right), 2 \cdot 33\left(s, 3 \mathrm{H}, \mathrm{CH}_{3}-10\right), 2 \cdot 5-2 \cdot 8\left(m, 6 \mathrm{H}, \mathrm{C}_{2}-3,5,6\right), 4 \cdot 6(t, 1 \mathrm{H}, \mathrm{CH}-2), 6 \cdot 95-$ $7 \cdot 5(d d, 2 \mathrm{H}, \mathrm{CH}-8,9)$. Analysis - Calculated for $\mathrm{C}_{15} \mathrm{H}_{16} \mathrm{O}_{2}: \mathrm{C}, 78.9 ; \mathrm{H}, 7 \cdot 0 \%$. Found: C, $78 \cdot 8 ; \mathrm{H}, 7 \cdot 15 \%$.

2,4-Dinitrophenylhydrazone derivative: Orange-red crystals from benzene, m.p. 222$23^{\circ} \mathrm{C}$. Analysis - Calculated for $\mathrm{C}_{21} \mathrm{H}_{20} \mathrm{~N}_{4} \mathrm{O}_{3}: \mathrm{N}, 13 \cdot 7 \%$. Found: $\mathrm{N}, 14 \cdot 05 \%$.

$R_{2}=R_{4}=H, R_{1}=R_{3}=R_{5}=\mathrm{CH}_{3}: 2,3,5,6$-Tetrahydro-2,7,10-trimethyl-4H-naphtho(I,2-b)pyran-4-one (5g): 5,8-Dimethyl-1-tetralone (Eisenbraun et al 1971) $(2 \mathrm{~g}$, $0.012 \mathrm{~mol})$ reacted with crotonic acid $(2 \mathrm{~g}, 0.024 \mathrm{~mol})$ in PPA $(20 \mathrm{~g})$. Elution in pet. ether(60-8.?):benzene $(70: 30)$ yielded the naphthopyrone as a red oil which was 
purified by bulb-to-bulb distillation $\left(198^{\circ} \mathrm{C} / 1 \mathrm{~mm}\right)$; yield $71 \%$. IR(neat) $\left(v, \mathrm{~cm}^{-1}\right): 1650$ $\mathrm{C}(\mathrm{C}=\mathrm{O}), \quad 1610,1595(\mathrm{Ar}) . \quad{ }^{1} \mathrm{H} \quad \mathrm{NMR}\left(80 \mathrm{MHz}_{2} \mathrm{CDCl}_{3}\right) \delta: 1 \cdot 57(d, J=6 \cdot 22 \mathrm{~Hz}, 3 \mathrm{H}$, $\left.\mathrm{CH}_{3}-2\right), 2 \cdot 25\left(\mathrm{~s}, 3 \mathrm{H}, \mathrm{CH}_{3}-7\right), 2 \cdot 34\left(\mathrm{~s}, 3 \mathrm{H}, \mathrm{CH}_{3}-10\right), 2 \cdot 47-2 \cdot 73\left(\mathrm{~m}, 6 \mathrm{H}, \mathrm{CH}_{2}-3,5,6\right)$, $4 \cdot 6(m, 1 \mathrm{H}, \mathrm{CH}-2), 6 \cdot 9-7 \cdot 4(m, 2 \mathrm{H}, \dot{\mathrm{C}} \mathrm{H}-8,9)$. Analysis - Calculated for $\mathrm{C}_{16} \mathrm{H}_{18} \mathrm{O}_{2}$ : C, $79 \cdot 3 ; \mathrm{H}, 7 \cdot 4 \%$. Found: C, $79 \cdot 5 ; \mathrm{H}, 7 \cdot 7 \%$.

2.4-Dinitrophenylhydrazone derivative: Red crystals from benzene, m.p. 219-20 $\mathrm{C}$, Analysis - Calculated for $\mathrm{C}_{22} \mathrm{H}_{22} \mathrm{~N}_{4} \mathrm{O}_{5}: \mathrm{N}, 13 \cdot 3 \%$. Found: $\mathrm{N}, 13 \cdot 5 \%$.

$R_{1}=R_{2}=R_{5}=H, \quad R_{3}=R_{4}=C_{3}:$ 7,9-Dimethyl-2,3,5,6-tetrahydro-4H-naphtho(1,2-b)pyran-4-one (5h): 5,7-Dimethyl-1-tetralone (Eisenbraun et al 1971) (2g, $0.012 \mathrm{~mol})$ reacted with acrylic acid $(1.8 \mathrm{~g}, 0.024 \mathrm{~mol})$ in PPA $(20 \mathrm{~g})$. Elution with pet. ether $(60-80)$ : benzene $(60: 40)$ afforded the naphthopyrone as a colourless oil which was purified by bulb-to-bulb distillation $\left(170^{\circ} \mathrm{C} / 1.5 \mathrm{~mm}\right)$, yield $68 \%$. IR (neat) $\left(v, \mathrm{~cm}^{-1}\right): 1665(\mathrm{C}=\mathrm{O}) ; 1595,1550$ (Ar). ${ }^{1} \mathrm{H} \quad \mathrm{NMR}\left(80 \mathrm{MHz} \mathrm{CDCl}_{3}\right) \delta: 2 \cdot 26$, $2 \cdot 34\left(s s, 6 \mathrm{H}, 2 \mathrm{CH}_{3}-5,7\right), 2 \cdot 4-2 \cdot 9\left(m, 6 \mathrm{H}, \mathrm{CH}_{2}-3,5,6\right), 4 \cdot 6\left(t, J=6.96 \mathrm{~Hz} .2 \mathrm{H}, \mathrm{CH}_{2}-\right.$ 2), $6 \cdot 85(s, 1 \mathrm{H}, \mathrm{CH}-8), 7 \cdot 0(s, 1 \mathrm{H}, \mathrm{CH}-10)$. Analysis - Calculated for $\mathrm{C}_{15} \mathrm{H}_{16} \mathrm{O}_{2}$ : C, $78.9 ; \mathrm{H}, 7.0 \%$. Found: C, 79.0; H, 7.2\%.

2,4-Dinitrophenylhydrazone derivative: Orange red crystals from benzene, m.p. 213$14^{\circ} \mathrm{C}$. Analysis - Calculated for $\mathrm{C}_{21} \mathrm{H}_{20} \mathrm{~N}_{4} \mathrm{O}_{5}: \mathrm{N}, 13 \cdot 7 \%$. Found: $\mathrm{N}, 13 \cdot 4 \%$

$R_{2}=R_{5}=H, \quad R_{1}=R_{3}=R_{4}=C_{3}: 2,3,5,6$ - Tetrahydro-2,7,9-trimethyl-4H-naphtho (1,2-b)pyran-4-one (5i): 5,7-Dimethyl-1-tetralone (Eisenbraun et al 1971) $(2 \mathrm{~g}$, $0.012 \mathrm{~mol})$ reacted with crotonic acid $(2 \mathrm{~g}, 0.024 \mathrm{~mol})$ in PPA $(20 \mathrm{~g})$. Elution with pet. ether (60-80): benzene (50:50) afforded red oil which was purified by bulb distillation $\left(187^{\circ} \mathrm{C} / 1.5 \mathrm{~mm}\right)$; yield $84 \%$. IR (neat) $\left(\mathrm{v}, \mathrm{cm}^{-1}\right): 1660(\mathrm{C}=\mathrm{O}), 1610$ (Ar). ${ }^{1} \mathrm{H}$ NMR $\left(80 \mathrm{MHz}, \mathrm{CDCl}_{3}\right) \delta: 1 \cdot 5\left(d, J=6 \cdot 2 \mathrm{~Hz}, 3 \mathrm{H}, \mathrm{CH}_{3}-2\right) ; 2 \cdot 24,2,23\left(s s, 6 \mathrm{H}, \mathrm{CH}_{3}-5,7\right), 2 \cdot 4-$ $2 \cdot 9\left(m, 6 \mathrm{H}, \mathrm{CH}_{2}-3,5,6\right), 4 \cdot 6(m, 1 \mathrm{H}, \mathrm{CH}-2), 6 \cdot 8(s, 1 \mathrm{H}, \mathrm{CH}-8), 7 \cdot 01(s, 1 \mathrm{H}, \mathrm{CH}-10)$. Analysis - Calculated for $\mathrm{C}_{16} \mathrm{H}_{18} \mathrm{O}_{2}: \mathrm{C}, 79 \cdot 3, \mathrm{H}, 7 \cdot 4 \%$. Found: $\mathrm{C}, 79 \cdot 6, \mathrm{H}, 7 \cdot 1 \%$.

2,4-Dinitrophenylhydrazone derivative: Orange red crystals from benzene, m.p. 232$33^{\circ} \mathrm{C}$. Analysis - Calculated for $\mathrm{C}_{22} \mathrm{H}_{22} \mathrm{~N}_{4} \mathrm{O}_{5}: \mathrm{N}, 13 \cdot 3 \%$. Found: $\mathrm{N}, 12 \cdot 9 \%$.

Dehydrogenation of 5 a to $4 \mathrm{H}$-naphtho(1,2,-b)pyran-4-one (8): $5 \mathrm{a}(100 \mathrm{mg}, 0.0005 \mathrm{~mol})$ was taken in diphenyl ether $(50 \mathrm{ml})$ to which was added $\mathrm{Pd} / \mathrm{C}(10 \%, 10 \mathrm{mg})$. The mixture was refluxed for $36 \mathrm{~h}$, cooled and filtered. The mixture was then subjected to steam distillation. The residue crystallised from pet. ether $(60-80)$ as pale brown crystals, m.p. $120-21^{\circ} \mathrm{C}(95 \mathrm{mg}, 96 \%)$. Further purification by sublimation $\left(140^{\circ} \mathrm{C}\right)$ in vaccuo $(2 \mathrm{~mm})$ gave colourless crystals, $123-24^{\circ} \mathrm{C}$ (lit. $\left.121-23^{\circ} \mathrm{C}\right)$. IR $(\mathrm{KBr})\left(v, \mathrm{~cm}^{-1}\right)$ : $1665(s, \mathrm{C}=\mathrm{O})$, Analysis - Calculated for $\mathrm{C}_{13} \mathrm{H}_{8} \mathrm{O}_{2}: \mathrm{C}, 79 \cdot 6 ; \mathrm{H}, 4 \cdot 1 \%$. Found: $\mathrm{C}, 79$; $\mathrm{H}, 4.4 \%$.

\section{Conclusion}

In summary, we have shown a simple and versatile method for the synthesis of naphtho(1,2-b)pyran-4-ones by condensation of various substituted 1-tetralones with $\alpha, \beta$-unsaturated acids such as acrylic and crotonic acids. Further, the same reaction can be used to synthesise the hitherto unknown spiro[naphthalene- 2,4 '-phenanthrene]-1, $1^{\prime}$-diones as the major product by proper control of stoichiometry and temperature of the reaction. 


\section{Acknowledgements}

We thank Dr K Nagarajan of Bangalore Pharmaceutical \& Research Laboratory, Bangalore and Dr S Y Dike of Alchemie Research Centre (ICI), Thane for valuable suggestions.

\section{References}

Ambrogini A, Passerini M 1976 Farmac, Edu. Sci. 31 627; 1976 Chem. Ahstr. 85192485

Annigeri A C, Siddappa S, Sirisi M 1966 J. Karnatak Univ. 11 59-63; 1967 Chem. Abstr. 67 2770t

De Barry B E and Sanders F G 1933 J. Chem. Soc. 434

Dean F M 1963 Naturally occurring oxygen ring compounds (London: Butterworths)

Eisenbraun E J, Hinman C W, Springer J M, Burnham J W and Chou T S 1971 J. Org. Chem. 362480

Evans R F and Smith J C 1954 J. Chem. Soc. 798

Kulkarni A B, Hosangadi B D and Kudav N A 1972 Some recent developments in the chemistry of natural products; (New Delhi: Prentice Hall of India)

Metz G 1972 Synthesis 614

Nohara A, Kuriki H, Saijo T, Sugihara H, Kanno M and Sanno Y 1977 J. Med. Chem. 20141

Nohara A, Umetani T and Sanno Y 1975 Japan Kokai 7529, 566; 1975 Chem. Abstr. 8379252

Olson C E and Bader A R 1963 In Organic synthesis-collective volume IV (ed.) (New York: John Wiley and Sons) p. 898

Ronald K W 1975 Br. Pat. 1, 373, 210; 1975 Chem. Abstr. 82 126615r

Rowlands D A 1985 In Synthetic reagents (ed.) J S Pizey (Chichester: Ellis Horwood) dvol. 6, pp. $164-67,175,185-87,219,243,261,314$

Schaffner-Sabba K, Schmidt-Ruppin K H, Wehrli W, Schuerch A R and Walsey J W F 1984 J. Med. Chem. 27990

von Strandtmann M, Klutchko K, Cohen M P and Shavel J Jr 1972 J. Heterocyclic Chem. 9171 